\title{
THE INTERSECTION OF THE SUBGROUPS OF FINITE INDEX IN SOME FINITELY PRESENTED GROUPS
}

\author{
RONALD HIRSHON
}

ABSTRACT. We consider the intersection of the subgroups of finite index in some finitely presented non-Hopfian groups.

The effort to establish the existence of a finitely generated non-Hopfian group seems to date back to the 1940's. R. Baer [5] was one of the first to consider this problem. Since then, several examples of finitely presented non-Hopfian groups have been given. If $G$ is any finitely generated group and if $\alpha$ is a surjective endomorphism of $G, \alpha$ induces in a natural way a surjective endomorphism $\bar{\alpha}$ of $G / N$, where $N$ is the intersection of the subgroups of finite index of $G$. But $G / N$ is residually finite and a finitely generated residually finite group is Hopfian. Hence $\bar{\alpha}$ is an automorphism and, consequently, the kernel of $\alpha$ is in $N$. Similarly if $K_{i}$ is the kernel of $\alpha^{i}$, $K_{i} \subset N$ so that if $K=\bigcup_{i \geq 1} K_{i}$, then $K \subset N$.

Professor W. Magnus has raised the question concerning under what circumstances $K=N$. The object of this note is to examine some of the known finitely presented non-Hopfian groups $G$ and to show that for these groups there exists an a such that $K$ is as large as possible, namely $K=N$.

Theorem. Consider the following groups $G$ with the respective endomorphisms $a$ :

(I) $\left\langle a, b ; a^{-1} b^{l} a=b^{m}\right\rangle, l, m$ relatively prime, $a \alpha=a, b a=b^{l}$;

(II) $\left\langle a, b, d ;[a, b, b]=[a, b, a]=[a, d]=1, a=\left[a^{2}, b, d\right]\right\rangle, a a=a^{2}$, $b a=b, d a=d ;$

$$
\text { (III) }\left\langle a, b, c ; a^{-1} c a=b^{-1} c b=c^{r}\right\rangle, r \geq 2, a \alpha=a, b a=b, c a=c^{r} \text {. }
$$

Then in each case above, $K=N$. In (I), $K=N=G^{\prime \prime}$.

Proof. For the proofs that a gives a surjective endomorphism which is not an automorphism, see [9], [7] and [18] respectively. To show $K=N$, it suffices to show $N \subset K$. Let $g \in N$. We consider (I). If $B$ is the normal subgroup generated by $b, G / B$ is residually finite so that $g \in B$. Hence we can express $g$ as a word in the elements $x_{i}=a^{i} b a^{-i}, g=w\left(x_{i}\right)$. Let $r$ be the largest positive integer such that $x_{r}$ appears in $w$. Let $-s$ be the smallest negative integer such that $x_{-s}$ appears in $w$. Let $\theta$ be the inner automor-

Received by the editor september 30, 1974 .

AMS (MOS) subject classifications (1970). Primary 20E25.

Key words and phrases. Hopfian group. 
phism: $u \rightarrow a^{-r} u a^{r}$. Then by using the relation $s_{1}^{l}=x_{i+1}^{m}$, one can easily check that $g \alpha^{r+s} \theta$ is a power of $b$. But $N \alpha=N, N \theta=N$ so $g a^{r+s} \theta$ is a power of $b$ in $N$. However, in any finitely generated group $G, N \subset G^{\prime \prime}$ since $G / G^{\prime \prime}$ is a residually finite group. Hence $g \alpha^{r+s} \theta=b^{j} \in G^{\prime \prime} \subset B^{\prime}$. However, $B / B^{\prime}$ is isomorphic to the additive group of rationals generated by $(l / m)^{i}$, $i=0,1,2, \ldots$, under the map $x_{i} B^{\prime} \rightarrow(l / m)^{i}[4, \mathrm{p} .477]$ so that $b^{j} \in B^{\prime}$ implies $j=0$. Hence $g \alpha^{r+s} \theta=1$ and, hence, $g \alpha^{r+s}=1$, so that $K=N$. To show that $N=G^{\prime \prime}$ it suffices to show that $G^{\prime \prime}$ is contained in any normal subgroup $L$ of finite index in $G$. But note that $a^{-k} b^{l k} a^{k}=b^{m^{k}}$ so that in any finite quotient group $\bar{G}$ of $G$, the order $j$ of $b$ is a divisor of $m^{k}-l^{k}$ for some $k$ so that $(j, l)=1$. Hence in this quotient group we may write $b=b^{s l}$ so that $a^{-1} b a=b^{m s}$. This implies that $\bar{G}$ has a cyclic commutator subgroup so that if $G / L=\bar{G}$, then $G^{\prime \prime} \subset L$.

For the group (II) we define $a_{0}=a$ and $a_{n+1}=\left(a_{n}, b, d\right)$. Now we assert the modulo $K$, generators for $A$, the normal subgroup generated by $a$, are $S=\left\{a_{n},\left(a_{n}, b\right)=c_{n}, n \geq 0\right\}$. For one set of generators of $A$ consists of the elements $w^{-1} a w$, where $w=w(b, d)$ is a freely reduced word in $b$ and $d$. We show by induction on the length of the word $w$ that $w^{-1}$ aw may be expressed as a product of elements in $S$ and elements in $K$. If the length of $w$ is zero, so that $w$ is the empty word, our assertion is apparent.

Assume the assertion is true for words of length $n$. Let $w$ be a word of length $n+1$. Write $w=u x$ where $u$ is of length $n$ and $x$ is either $b, d$, $b^{-1}$ or $d^{-1}$. Then by our inductive assumption,

$$
u^{-1} a u=s_{1}^{e}{ }_{1} s_{2}^{e} \ldots s_{j}^{e} j_{k,}, \quad e_{i}= \pm 1
$$

for some $s_{i} \in S$ and $k \in K$. Hence $w^{-1} a w=\left(x^{-1} s_{1} x\right) \ldots\left(x^{-1} s_{j} x\right)\left(x^{-1} k x\right)$. Now since $K$ is a normal subgroup, $x^{-1} k x \in K$. Hence, we need only show that if $x \in S, x^{-1} s x$ may be expressed as a product of elements $s_{*}, s_{*}^{-1}$, $k_{*}$ where $s_{*} \in S, k_{*} \in K$.

We first consider $s=a_{n}$. Note $a_{n} \alpha^{n}=a$. Hence, $\left(d, a_{n}\right) \alpha^{n}=(d, a)=1$, so that $d^{-1} a_{n} d=a_{n} \bmod K$ and $d a_{n} d^{-1}=a_{n} \bmod K$. Also $b a_{n} b^{-1}=$ $\left(b^{-1}, a_{n}^{-1}\right) a_{n}$. However, $\left[\left(b^{-1}, a_{n}^{-1}\right) c_{n}\right] a^{n-1}=1$, so that $\left(b^{-1}, a_{n}^{n}\right)=c_{n}^{-1}$ $\bmod K$, so that $b a_{n} b^{-1}=c_{n}^{-1} a_{n} \bmod K$. Similarly, $b^{-1} a_{n} b=c_{n} a_{n} \bmod K$. Now to consider $s=c_{n}$, note

$$
d^{-1} c_{n} d=\left(d, c_{n}^{-1}\right) c_{n} .
$$

But we claim

$$
\left(d, c_{n}^{-1}\right)=\left(d, c_{n}\right)^{-1} \bmod K .
$$

To see this, note that if $\bar{a}=b^{2} d^{-1}(a, b) d b^{-2}$, then a brief calculation shows $\bar{a}^{2}=a[7, \mathrm{p}, 197]$ so that $\bar{a} \alpha=\bar{a}^{2}=a$. 
Now, note that $c_{0}^{-1} d c_{0}=d \bar{a} \bmod K$ and $(d, \bar{a})=1 \bmod K$. This implies that $\left(d, c_{0}\right)^{-1}=\left(d, c_{0}^{-1}\right) \bmod K$. Now if we apply $\alpha^{n}$ to $\left(d, c_{n}\right)$ and $\left(d, c_{n}^{-1}\right)$, respectively, we may deduce (2), so that from (1) and (2) we see $d^{-1} c_{n} d=$ $a_{n+1} c_{n} \bmod K$. Similarly $d c_{n} d^{-1}=a_{n+1}^{-1} c_{n} \bmod K$. Clearly, $b^{-1} c_{n} b=c_{n}$ mod $K$ so that our assertion about $S$ is valid.

Now note that $G / A$ is a free group of rank two which is consequently residually finite. This implies that $N \subset A$. Hence if $w \in N$, we may write $w=s_{1}^{\epsilon_{1}} s_{2}^{\epsilon_{2}} \ldots s_{j}^{\epsilon_{j}} k$, where $s_{i} \in S$ and $k \in K$. Note that $a_{n} a^{n}=a, c_{n} a^{n}=$ $(a, b), n \geq 1$, so that we may find a positive integer $r$ such that $w \alpha^{r}=$ $a^{j}(a, b)^{-m} \in N$. Hence in every finite quotient $\bar{G}$ of $G$ we have $a^{j}=(a, b)^{m}$. Hence in $\bar{G},\left(a^{j}, b\right)=(a, b)^{j}=1$. Hence in $\bar{G}, a^{j}=\left(c_{0}^{2 j}, d\right)=1$ and $a^{m}=$ $\left(c_{0}^{2 m}, d\right)=1$. Hence in $G, a^{j} \in N$ and $a^{m} \in N$. We will show this implies $j=m=0$ so that $w \alpha^{r}=0$. To do this, for odd $n$ let $L_{n}$ be the normal subgroup of $G$ generated by $a^{n^{2}}, b^{n^{2}}, d^{n^{2}}, n>1$. Let

$$
\begin{aligned}
& P_{n}=\left\langle a, b ;(a, b, a)=(a, b, b)=a^{n^{2}}=b^{n^{2}}=1\right\rangle, \\
& M_{n}=\left\langle c, d ;(c, d, c)=(c, d, d)=c^{n^{2}}=d^{n^{2}}=1\right\rangle .
\end{aligned}
$$

Let $H_{n}$ be the subgroup of $P_{n}$ generated by $\left(a^{2}, b\right)$ and $a$. Let $J_{n}$ be the subgroup of $M_{n}$ generated by $(c, d)$ and $c$.

Form the free product of $P_{n}$ and $M_{n}$ amalgamating $H_{n}$ and $J_{n}$ as $c=$ $\left(a^{2}, b\right)$ and $a=(c, d)$. This free product with $H_{n}=J_{n}$ is just $G / L_{n}$. Now a free product of finite groups with a subgroup amalgamated is residually finite. If $n>1$ is odd, $a^{n} \neq 1$ in $G / L_{n}$ so that we can find a finite quotient of $G / L_{n}$ (and hence of $G$ ) in which $a^{n} \neq 1$. Hence, if $t$ is odd, $a^{t} \notin N$ in $G$. Moreover, if $t$ is even, $t \neq 0, t=2^{q} f$, $f$ odd, then $a^{t} \notin N$, for as can be checked from the defining relations of $G$, the order of $a$ in every finite quotient of $G$ is odd so that $a^{t} \in N$ would imply $a^{f} \in N$.

For the group (III), if $C$ is the normal subgroup generated by $c$, we see that $G / C$ is a free group of rank two so that $N \subset C$. Moreover, generators for $C$ are $w^{-1} c w$, where $w=w(a, b)$ is a freely reduced word in $a$ and $b$. Now we claim that modulo $K$ generators of $C$ are the elements $c_{j}=a^{j} c a^{-j}$, $j \geq 0$. To show this we show by induction on the length of the word $w$ that any element $w^{-1} c w$ is expressible in terms of the $c_{j}$ and elements of $K$. If the length of $w$ is zero, that is, if $w$ is the empty word, this is clear. To complete the induction it suffices, as in the discussion for the group (II), to show that $x^{-1} c_{j} x$ is expressible in terms of the $c_{t}$ and elements in $K$, where $x$ is one of the elements $a, b, a^{-1}, b^{-1}$. To this end note that $a c_{j} a^{-1}=$ $c_{j+1}$ and $a^{-1} c_{j} a=c_{j-1}$ if $j \geq 1$ while $a^{-1} c_{0} a=c_{0}^{r}$. Also we note that if $b_{j}=b^{j} c b^{-1}, j \geq 0$, then $b_{j}=a_{j} \bmod K$. For note, $b_{j} a=b^{j} c^{r} b^{-j}=b^{j} b^{-1} c b b^{-j}$ $=b_{j-1}, j \geq 1$. Hence $b_{j} a^{j}=b_{0}=c$. Similarly $c_{j} a^{j}=c$ so that $\left(b_{j} c_{j}^{-1}\right) a^{j}=$ 
1. Hence modulo $K$ we may write $b c_{j} b^{-1}=b b_{j} b^{-1}=b_{j+1}=c_{j+1}$ and

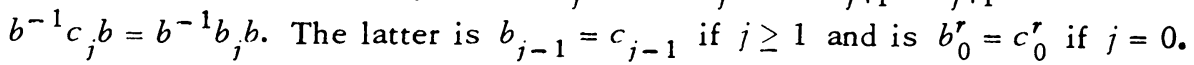
This completes the proof that elements of $N$ are expressible in terms of the $c_{j}$ and elements in $K$.

In view of the fact that $c_{j} a^{j}=c$, it follows that if $w \in N$, then $w \alpha^{t}=$ $c^{u}$ for some positive integer $t$. Hence $c^{u} \in N$. However, if we consider the groups

$$
\begin{aligned}
& P_{n}=\left\langle a, c ; a^{-1} c a=c^{r}, a^{n}=1\right\rangle, \quad n \geq 2, \\
& M_{n}=\left\langle b, d ; b^{-1} d b=d^{r}, b^{n}=1\right\rangle, \quad n \geq 2,
\end{aligned}
$$

then $c$ and $d$ have the same orders and $c^{n} \neq 1$. Since $P_{n}$ and $M_{n}$ are finite groups, their free product with the amalgamation $c=d$ is residually finite. However this free product yields a homomorphic image of $G$. Hence if $c^{u} \epsilon$ $N$ in $G$, we must have $u=0$.

Problem. Does every finitely presented non-Hopfian group have a surjective endomorphism a for which $K=N$ ?

Some of the references listed below are not referred to in the present paper but are relevant to the topic of Hopficity. References for any assertions made about residually finite groups can be found in the survey paper [30].

In conclusion, the author expresses his deep gratitude to Professor Wilhelm Magnus for suggesting to him that the results of this note were true and for his encouragement to pursue the details and for the several valuable conversations during the writing of this note.

\section{REFERENCES}

1. Michael Anshel, Non-Hopfian groups with fully invariant kernels, Ph. D. Dissertation, Adelphi University, 1967.

2. The endomorphisms of certain one-relator groups and the generalized Hopfian problem, Bull. Amer. Math. Soc. 77 (1971), 348-350. MR 42 \#7757.

3. - Non-Hopfian groups with fully invariant kernels. I, Trans. Amer. Math. Soc. 170·(1972), 231-237. MR 46 \#3626.

4. - Non-Hopfian groups with fully invariant kernels. II, J. Algebra 24 (1973), 473-485. MR 47 \#1960.

5. R. Baer, Groups without proper isomorphic quotient groups, Bull. Amer. Math. Soc. 50 (1944), 267-278. MR 5, 228.

6. G. Baumslag, Hopficity and abelian groups, Topics in Abelian Groups (Proc. Sympos., New Mexico State Univ., 1962), Scott, Foresman, Chicago, Ill., 1963, pp. 331-335. MR $30 \# 139$.

7. - A non-Hopfian group, Bull. Amer. Math. Soc. 73 (1967), 403-418.

8. - On abelian Hopfian groups. I, Math. Z. 78 (1962), 53-54. MR 26 $\# 202$.

9. G. Baumslag and D. Solitar, Some two-generator one-relator non-Hopfian groups, Bull. Amer. Math. Soc. 68 (1962), 199-201. MR 26 \#204.

10. R. G. Burns, On the question of the Hopficity of the direct product of a Hopfian group with an infinite cyclic group, Arch. Math. 24 (1973), 579-581.

11. A. L. S. Corner, Three examples on Hopficity in torsion-free abelian groups, 
Acta Math. Acad. Sci. Hungar. 16 (1965), 303-310. MR 32 \#1253.

12. I. M. S. Dey, Free products of Hopf groups, Math. Z. 85 (1964), 274-284. MR $30 \# 140$.

13. - Embeddings in non-Hopf groups, J. London Math. Soc. (2) 1 (1969), 745-749. MR 40 \#377.

14. S. Dick, Dual-Hopfian abelian groups, $\mathrm{Ph}$. D. Dissertation, Adelphi University, June 1968. $18-20$.

15. M. J. Dunwoody, The Hopficity of $F / R$, Bull. London Math. Soc. 3 (1971),

16. K. N. Frederick, The Hopfian property for a class of fundamental groups, Comm. Pure Appl. Math. 16 (1963), 1-8. MR 26 \#6948.

17. G. Gratzer and H. Sichler, Free products of Hopfian lattices, J. Austral. Math. Soc. 17 (1974), 234-246.

18. G. Higman, A finitely related group with an isomorphic proper factor group, J. London Math. Soc. 26 (1951), 59-61. MR 12, 390.

19. R. Hirshon, Some results on direct products of Hopfian groups, Ph. D. Dissertation, Adelphi University, New York 1967.

20. - Some theorems on Hopficity, Trans. Amer. Math. Soc. 141 (1969), 229-244. MR 41 \#3584. \#8513.

21. - On Hopfian groups, Pacific J. Math. 32 (1970), 753-766. MR 41

22. - The center and commutator subgroup in Hopfian groups, Ark. Mat.

9 (1971), 181-192. MR 47 \#8701.

23. - A conjecture on Hopficity and related results, Arch. Math. (Basel) 32 (1971), 429-455. MR $46 \# 261$.

24. Some new groups admitting essentially unique directly indecomposable decompositions, Niath. Ann. 194 (1971), 123-125. MR 44 \#4100.

25. - The direct product of a Hopfian group with a group with cyclic center, Ark. Mat. 10 (1972), 231-234. MR $47 \# 8702$. 380 .

26. - A problem in group theory, Amer. Math. Monthly 79 (1972), 379-

27. The direct product of a Hopfian group with a p-group, Arch. Math. (to appear).

28. - The cancellation of an infinite cyclic group in direct products Arch. Math. 26 (1975), 134-138.

29. J. M. Tyrer Jones, Direct products and the Hopf property, J. Austral. Math. Soc. 17 (1974), 174-197.

30. W. Magnus, Residually finite groups, Bull. Amer. Math. Soc. 75 (1969), 305316. MR $39 \# 2865$.

31. B. H. Neumann, A two-generator group isomorphic to a proper factor group, J. London Math. Soc. 25 (1950), 247-248. MR 12, 390.

32. H. Neumann and I. M. S. Dey, The Hopf property of free products, Math. Z. 117 (1970), 325-339. MR 43 \#2099.

33. M. F. Newman and G. E. Wall, Hanna Neumann, J. Austral. Math. Soc. 17 (1974), 1-29.

34. M. Orzech, Onto endomorphisms are isomorphisms, Amcr. Math. Monthly 78 (1971), 357-362. MR $43 \# 6195$.

35. M. Orzech and L. Ribes, Residual finiteness and the Hopf property in rings, J. Algebra 15 (1970), 81-88. MR $41 \# 248$.

36. P. Schupp and C. Miller, Embeddings into Hopfian groups, J. Algebra (to appear).

37. K. Seksenbaev, The Hopficity of a wreath product of two groups, lzv. Akad. Nauk Kazah. SSR Ser. Fiz.-Mat. Nauk 1971, no. 3, 50-54. (Russian) MR 45 \#6932.

DEPARTMENT OF MATHEMATICS, POLYTECHNIC INSTITUTE OF NEW YORK, BROOKLYN, NEW YORK 11201 